\title{
A study of impact of post-retirement work on psychological well- being of elderly
}

\author{
Rina Pranay Patel \\ Assistant professor, Department of Psychology, Bhavan's H. Somani College, Mumbai \\ Corresponding author: Ms. Rina Patel \\ Email-rinadhonde@yahoo.com
}

\begin{abstract}
Background: Elderly people are important aspect of our society. Their experience and wisdom are necessary for the society to grow. Hence, they need to be preserved with care. Studies show that being active has a positive correlation with mental health. Work provides such an opportunity for being active. However, it is a general observation that retirement brings a change in the daily routine of the elderly and thereby reduces the amount of activities. This paper is an attempt to find if working after retirement has an impact on psychological well-being of the elderly.

Methods: This paper compares 20 elderly working post-retirement and 20 elderly not working postretirement on their psychological well-being. Ryff's Psychological well-being scale is used to measure the psychological well-being of the elderly. Data is collected from the selected sample and test is conducted on the data.

Results: The $t$ value for the data is $5.37(\mathrm{p}<0.01)$. Thus the results show that elderly involved in postretirement work have higher psychological well-being than those not involved in post-retirement work.

Conclusion: Working post retirement is a positive factor in maintaining psychological well being in the elderly.
\end{abstract}

Keywords: Elderly, post-retirement work, psychological well-being.

(Paper received $-2^{\text {nd }}$ January 2018, Peer review completed $-10^{\text {th }}$ January 2018)

(Accepted $-16^{\text {th }}$ January 2018)

\section{INTRODUCTION}

Aging is a global phenomenon. It is no longer limited to any industrialized societies. The -population of the elderly is growing everywhere. The world is experiencing an actual demographic graying of the planet [1]. India's population is likely to rise in the next 50 years and the number of elders above age 60 will shoot dramatically. India has 100 million elderly today [2] and this figure will raise constituting $20 \%$ of India's total population by 2050. Advance medical treatment and economic well-being and good health care facilities has brought down the mortality rate of the elderly. As the number of elderly grows up so will the issues related to old age go up. Hence government needs to plan policies for this growing elderly.

Government of India adopted 'National Policy on Older Persons 'in January 1999 which defines 'senior citizen' or 'elderly' as a person who is of age 60 years or above. Ageing is not biological but a social and psychological process as well. Reducing physical capacities, changing role in the family and society, financial dependency of children and lack of opportunities to use their knowledge and wisdom can bring new challenges in front of the elderly. A lot of unutilized free time can add on to this challenge. Hence readjustment with family and society and channelizing their resources is essential. 
One major change is brought about in the life of elderly due to retirement from work. Work provides us with inner joy of achieving something. It saves us from dullness and boredom of life and helps channelize our energy. Retirement not only leads to financial crunches but also creates hollowness in the life of the elderly. Thus, retirement is a major transition in the life of an individual. Different people react differently to retirement. Thoughts of Retirement often lead to concerns about financial issues and about spending hours of relaxation. Webster defines retirement as "withdrawal from active engagement in one's occupation or profession." However, in fact it is much more. Retirement is an identity shift for many people. It is a process, not an event. Thus retirement, like any transition in a person's life, has both emotional rewards and hazards.

Various aspects in persons' life both pre and post-retirement can determine their adjustment to it. One such important aspect in the post-retirement life is the kind of activities in which elderly involve themselves. Staying connected with others is an important benefit of work. Co-workers play an important role in maintaining social relationships. Whether a person is innately 'Social' or not, everyone requires social activities that connect them to others in life. However, retirement can bring a loss of such connection with people. Activities that involve other people can allow elderly to maintain social contacts even after retirement. Such activities may include volunteering in some projects, helping people, educating others, involving in social work, pursuing hobbies and interests, etc. Such activities can be even converted into taking up a part time or full time job after retirement. Studies show that individuals who continue to engage themselves in some kind of activities are generally happier than those who don't. Such activities have an impact on the psychological well-being of the elderly.

Psychological well-being is the general level of happiness and satisfaction of a person. It refers to how people evaluate their lives. Ryff proposed a 'Six-factor model of Psychological Well-being'. Ryff has noted six core dimensions in her model. These dimensions include Autonomy, Environmental mastery, Purpose in life, Positive relations with others, Personal growth and Self-acceptance [3]. Autonomy is the degree to which the individual is self determining and independent. Environmental master is the degree of mastery and competence in managing the environment. Personal growth is the degree of experiencing continued growth in life. Positive relation with others is the degree to which individual has satisfying relations in life. Purpose in life is the degree to which individual has goals and meaning in life. Self acceptance is the attitude that individual has towards themselves and how they accept their past and present.

The present study is an attempt to compare the psychological well-being of the elderly who are involved in some kind of post- retirement work and that of the elderly who are not involved in any kind of postretirement work.

A lot of Research has been done with respect to Psychological well-being in the elderly. Research has focused on activities performed post-retirement as an indicator of high psychological well- being.

Osborne conducted a study on Psychological effects of disengagement from a work life and the transition to retirement. According to him disengagement from work results in various problems like partial identity disruption, lack of ability to make decisions, search for engagement in society, lowered self trust, etc. Some aspects of preretirement life that can predict a successful transition to retirement are also presented [4].

$\mathrm{Wu}$ and others conducted a study on 501 Chinese elderly who were either volunteers or non-volunteers. $65 \%$ of them were involved in community voluntary work for an average of four hours per week from their retirement. As compared to the non-volunteers, volunteers reported better physical health, greater life satisfaction, higher self efficacy and lower psychological distress [5].

Kim and Moen in their study investigated the relationship between retirement transition and psychological well-being using data of 458 married men and women aged 50-72 who were either in their career, retired or had just made transition to retirement [6]. Results show that relationship between retirement and psychological well-being has a temporal, life course context. They suggested that various resources and context surrounding retirement transition are important in understanding the relationship between retirement transition and psychological well-being. 


\section{METHODOLOGY}

\section{Hypothesis}

Elderly engaged in post-retirement work will be significantly higher on Psychological well-being as compared to those not engaged in post-retirement work.

\section{Operational Definition of Variables \\ Independent Variable}

Kind of Post-retirement work as categorized on the criteria of time spent per week, goals, income, work schedule, experience and expertise needed and the duration for which they are engaged in work.

a. Elderly involved in post-retirement work - These include elderly who have taken up a work or job in formal organizations, small firm, Consultancy services, working as an Adjunct faculty in some Institute, etc. These activities demands time and resources. A person must spend a minimum of 30 hours per week in such work. Such activities will require experience and formal expertise. These activities provide a structure to post-retirement life and are a source of earning for the individual.

b. Elderly not involved in post-retirement work - These involved elderly who are not engaged in any kind of post-retirement work. The individuals may involve themselves in any kind of casual work to spend their time. This may include helping in household activities, child care, watching television, reading books, gardening, trekking, religious activities, etc. There is no time constraint for such work and is not a source of income. Such activities will require no experience and formal expertise.

\section{Dependent Variable}

Psychological well-being - It is the way people evaluate their life and happiness. Psychological well-being is based on the Ryff's Model which explains six aspects of psychological well-being as measured by Ryff Scale of Psychological well-being.

a. Autonomy - It is the degree to which the individual is self determining and independent.

b. Environmental mastery - It is the degree of mastery and competence in managing the environment.

c. Personal growth - It is the degree of experiencing continued growth in life.

d. Positive relation with others - It is the degree to which individual has satisfying relations in life.

e. Purpose in life - It is the degree to which individual has goals and meaning in life.

f. Self acceptance - Attitudes individual have towards themselves and how they accept their past and present.

\section{Controlled variables}

The geographical areas, socioeconomic status, medical conditions, retirement condition (no voluntary retirement) are the variables that are controlled in the study.

\section{Design}

A Comparative research design is used to describe the relationship of Psychological well-being, with Postretirement work.

\section{Sample}

A non-probability purposive sample of 40 retired persons from the city and suburbs of Mumbai, falling in the age group 65-70 years, 20 each in the two groups of Elderly involved in post-retirement work and Elderly not involved in post-retirement work are taken for the study. The sample constitutes of Elderly who were holding Class I or Class II positions in Government or Semi- Government Organizations or equivalent posts in the Corporate or Private sector. The male and female ratio is approximately 2:1. The educational qualification of the sample is minimum higher secondary schooling. The sample belongs to middle socioeconomic status. Elderly with work experience of minimum 20 years prior to the retirement and are working for a minimum of three years post-retirement are considered for the study. Elderly who are psychologically and physically fit are taken as sample. 


\section{Instruments}

1. Demographic Information sheet- An information sheet to collect demographic information like age, qualification, experience of work, medical information and years of involvement in Postretirement work is used.

2. Ryff Scale of Psychological Well-being (RSPWB) - It measures the aspects of Psychological well-being. It is developed by Researcher Carol Ryff. The medium form of Ryff Scale of Psychological well-being consisting of 54 items in English is used for the study. It measures 6 aspects of psychological well-being viz. autonomy, environmental mastery, personal growth, positive relations with others, purpose in life and self acceptance. Respondent rate the items on a scale of 1 to 6 with 1 indicating strong disagreement and 6 indicating strong agreement. Respondents are totalled for each of the six categories. About half the items have reverse scoring. High score in each category indicates mastery in that area of life. The scores on six categories can then be combined to get one single score on Psychological well-being. The internal consistency of all six subscales range from 0.86 to 0.93 . The test-retest reliability of the subscales range from 0.81 to 0.88 . The validity studies show that there is a negative correlation between psychological wellbeing and severity of psychological distress measured one year apart. [7]. The correlations with prior measures of positive functioning (i.e., life satisfaction, affect balance, self esteem, internal control, and morale) are all positive and significant, with coefficients ranging from .25 to .73. Similarly, correlations with prior measures of negative functioning (i.e., powerful others, chance control, depression) are all negative and significant, with coefficients ranging from -.30 to -.60.

\section{Data collection and analysis}

Data is collected by initially approaching approximately 50 elderly and by screening them on the defined criteria, 20 each are then selected in the two groups of the Independent variable by following the standard procedures of data collection. Their informed consent is taken. Psychological instruments are then administered after taking their demographic details. Descriptive statistics such as mean, standard deviation and $t$ test is conducted on the sample.

\section{RESULTS}

The results show that there is significant difference between the two groups on their psychological wellbeing. The mean of the scores for psychological well-being of the elderly involved in post-retirement work is 205.25 and that of the elderly not involved in post-retirement work is 163.35 . The $t$ value is 5.37 ( $\mathrm{p}<$ $0.01)$

\section{DISCUSSION}

Retirement is a major milestone in the life of every individual particularly for those who have spent a major part of their life doing a job. Retirement may bring a sudden emptiness in their extremely occupied life. When work provides with a lot of social connectedness, retirement may break these social ties and may bring loneliness. Especially with the nuclear family set up the elderly have few people with whom they can share or even talk at times. Work gives opportunity to utilize one's knowledge and creativity which retirement may take away. Thus, retirement can be a real challenge if it is not properly planned. It may lead to various physical problems and psychological distress if not dealt with properly. Postretirement work provides a buffer to the negative impact of retirement on the elderly. The current study proves that post-retirement work enhances psychological well-being of elderly.

The hypotheses that states that the elderly engaged in post-retirement work will be significantly higher on psychological well-being as compared to those not engaged in Post-retirement work is validated by the data. This may be because of the benefits that the post-retirement work offers to the elderly. Continuing to work after retirement reduces the feeling of being dependent for their daily needs on their children who were dependent on them at one point of time. Work keeps them engaged in some constructive activity 
which helps them distract from their physical discomforts. As described by Wu A.M. et. al. in their study, post-retirement work enhances self-efficacy and increases life satisfaction. Also the study conducted by Sneed R.S. and Cohen S. (2013) found a positive correlation between psychological well-being and volunteering activities $[5,8]$.

\section{CONCLUSION}

The hypothesis is validated by the data. It is thus proved by the data that involvement in post-retirement work has a positive impact on psychological well-being. Involving oneself in some constructive work increases the sense of autonomy and thereby increases one's control over their environment. Work provides with a purpose for living. It enhances the positive relation with others and helps the individual to deal with their life issues in a better manner. More than anything, work gives a sense of achievement and a continued personal growth to the elderly. All this may reduce the anxiety and feelings of negativity in their life. However such involvement in post- retirement work should be voluntary and not forced by circumstances or else it may also have negative impact.

\section{Limitation of the study}

- The sample size is small.

- The sample is taken only from the central suburbs of Mumbai.

- All variables in the family and work life of the elderly are not taken into account.

\section{REFERENCES}

1. Galambos C, Rosen A. The aging are coming and they are us. Health Soc Work 1999;24(1):73-9.

2. American Psychological Association. Guidelines for Psychological practices with older adults. APA; 2004.

3. Ryff $\mathrm{CD}$. Happiness is everything, or is it? Explorations on the meaning of psychological well-being. J Personal Soc Psychol 1989;57(6):1069.

4. Osborne JW. Psychological Effects of the Transition to Retirement/Effets psychologiques de la transition vers la retraite. Can J Couns Psychother (Online) 2012;46(1):45-9.

5. Wu AM, Tang CS, Yan EC. Post-retirement voluntary work and psychological functioning among older Chinese in Hong Kong. J Cross-Cultural Gerontol 2005;20(1):27-45.

6. Kim JE, Moen P. Retirement transitions, gender, and psychological well-being: A life-course, ecological model. J Gerontol Series B: Psychol Sci Soc Sci 2002;57(3):P212-22.

7. Abbott RA, Ploubidis GB, Huppert FA, Kuh D, Wadsworth ME, Croudace TJ. Psychometric evaluation and predictive validity of Ryff's psychological well-being items in a UK birth cohort sample of women. Health Qual Life Outcomes 2006;4(1):76-82.

8. Sneed RS, Cohen S. A prospective study of volunteerism and hypertension risk in older adults. Psychol Aging 201328(2):578-86.

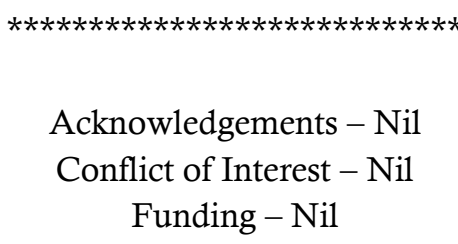

